\title{
A method for volumetric visualization of temperature distribution: three-dimensional meshed infrared thermography
}

DOI:

10.1007/s00348-019-2704-7

\section{Document Version}

Accepted author manuscript

Link to publication record in Manchester Research Explorer

Citation for published version (APA):

Ozer, O., Kumlutas, D., \& Yucekaya, U. A. (2019). A method for volumetric visualization of temperature distribution: three-dimensional meshed infrared thermography. Experiments in Fluids, 60(4). https://doi.org/10.1007/s00348-019-2704-7

\section{Published in:}

Experiments in Fluids

\section{Citing this paper}

Please note that where the full-text provided on Manchester Research Explorer is the Author Accepted Manuscript or Proof version this may differ from the final Published version. If citing, it is advised that you check and use the publisher's definitive version.

\section{General rights}

Copyright and moral rights for the publications made accessible in the Research Explorer are retained by the authors and/or other copyright owners and it is a condition of accessing publications that users recognise and abide by the legal requirements associated with these rights.

\section{Takedown policy}

If you believe that this document breaches copyright please refer to the University of Manchester's Takedown Procedures [http://man.ac.uk/04Y6Bo] or contact uml.scholarlycommunications@manchester.ac.uk providing relevant details, so we can investigate your claim.

\section{OPEN ACCESS}




\title{
A Method for Volumetric Visualization of Temperature Distribution: Three-Dimensional Meshed Infrared Thermography
}

\author{
Özgün Özer*, Dilek Kumlutaş, Utku Alp Yücekaya \\ Mechanical Engineering Department, Dokuz Eylul University, Tınaztepe, Izmir, Turkey. \\ *Corresponding author. Tel.:+90 5384328681 E-mail address: ozgunozer@gmail.com
}

\begin{abstract}
In this study, a method called three-dimensional meshed infrared thermography (3D MIT) was developed to visualize the volumetric temperature of air using an infrared camera. The main operating principle of the proposed method is to image the spheres using an infrared camera and processing the images with computer software to obtain the volumetric temperature distribution. For the correct implementation of the method, an equation is proposed to determine the distance between the thermal target and the measurement target placed in the flow to be examined. The proposed method was compared with conventional measurement-screen methods, namely those using a plane target and a high porosity target, via particle image velocimetry (PIV) in terms of flow effects. The temperature measurement capability of the proposed method is presented in comparison with the results of thermocouple and conventional measurement-screen-based measurements recorded using a jet flow. In addition, the volumetric temperature isosurfaces obtained via the 3D MIT method of a jet flow were compared with the volumetric velocity isosurfaces obtained via the PIV method in terms of the flow structure.
\end{abstract}




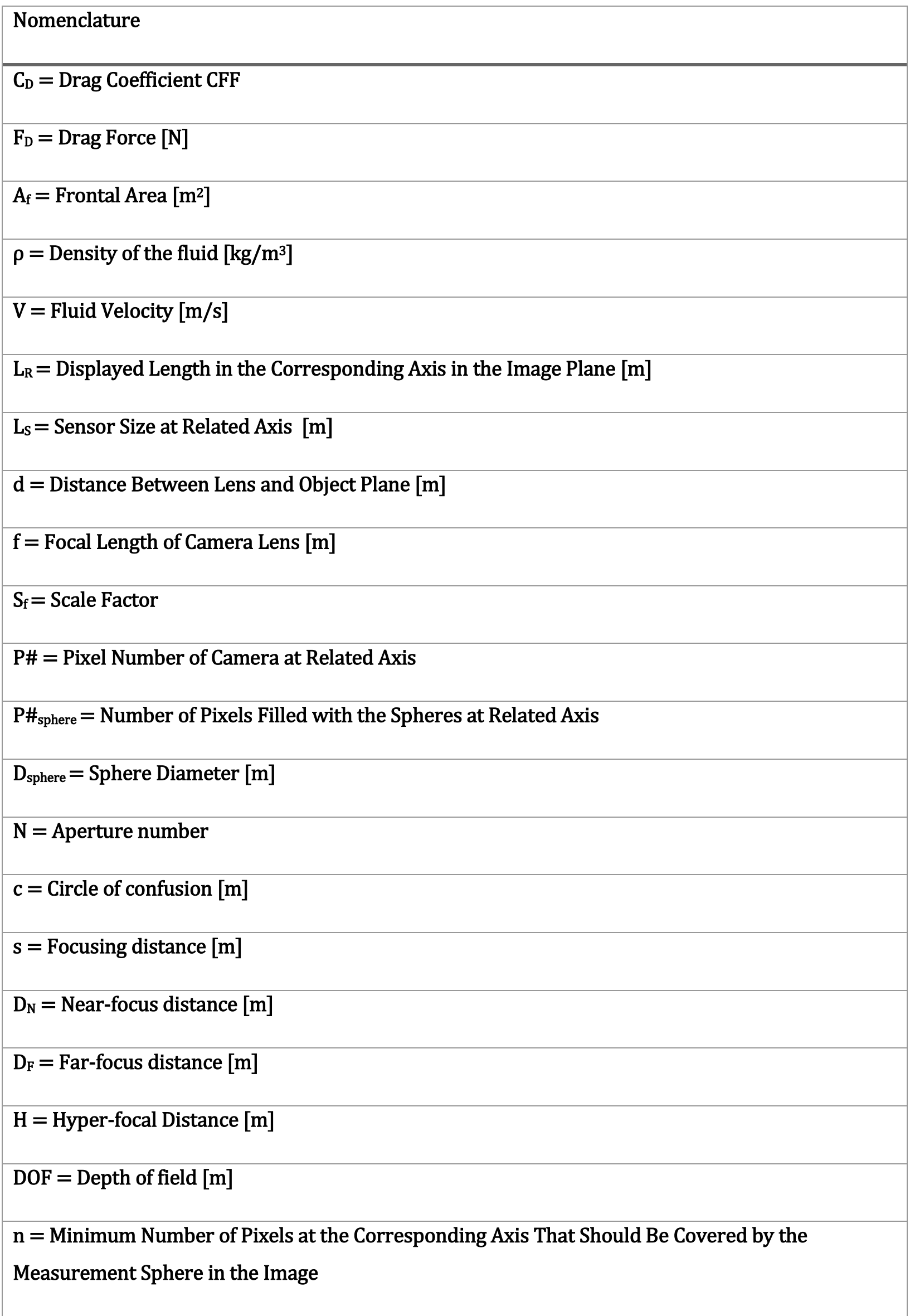




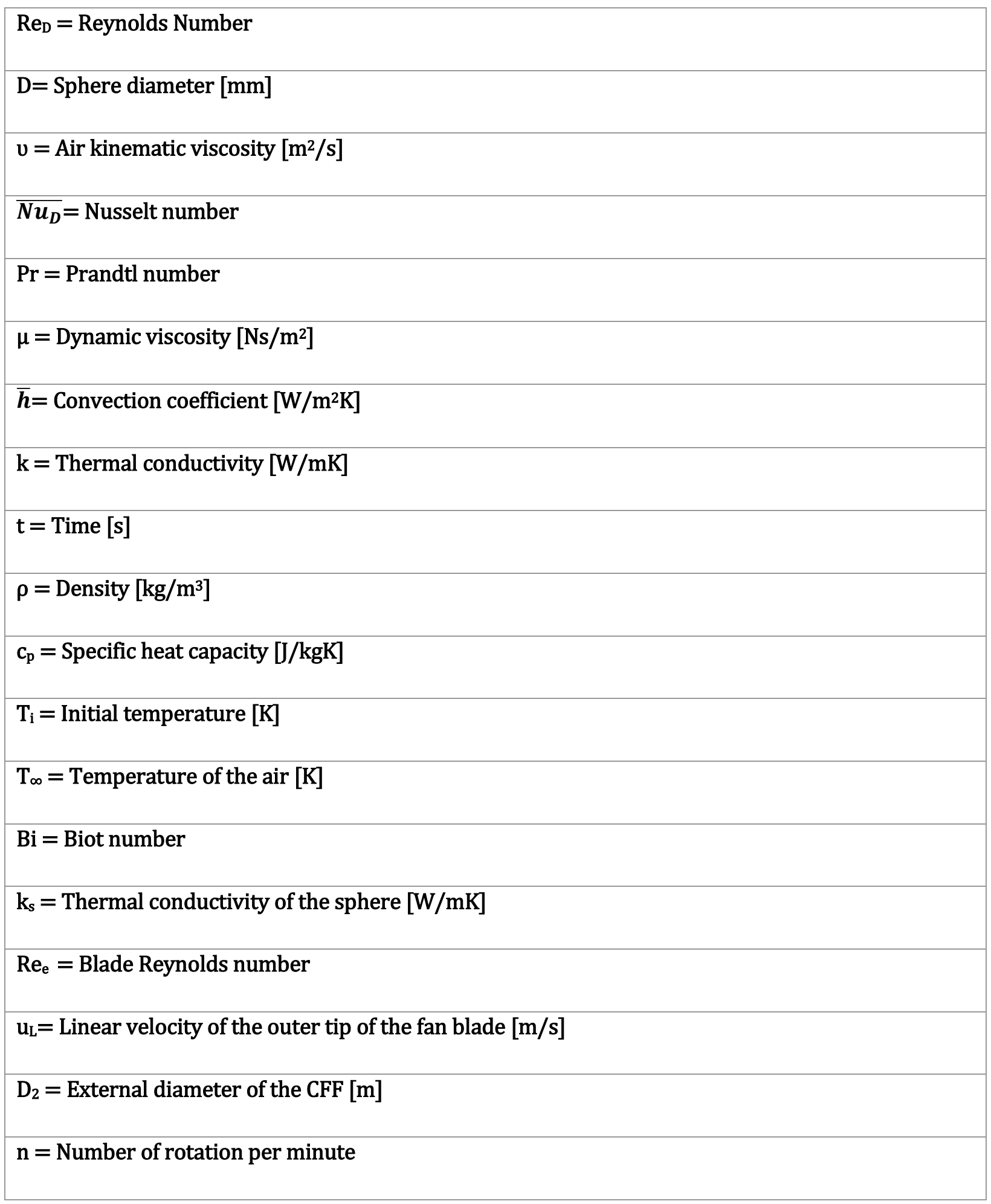

\section{1-Introduction}

Infrared imaging techniques are currently used for determining the temperature of solid materials that are opaque in infrared wavelengths and radiate with a distinct emissivity coefficient. The gases that have high temperature and high particle density can be visualized 
with infrared cameras. However, owing to the fact that gases are transparent at infrared wavelengths $(7-13 \mu \mathrm{m})$, low signal to noise ratio and unknown emissivity distributions, infrared cameras cannot determine the temperature of gases directly. In the literature, measurement screens have been used in order to solve this problem.

A measurement screen is a solid target that is positioned in a gas medium and allows us to determine the temperature of the gas via convection. The temperature distribution of the measurement screen is obtained via imaging with infrared thermography. It should be noted that this method measures the temperature of gases indirectly; therefore, the success of the measurement depends on the mechanism of heat transfer between the measurement screen and the gas. From this standpoint, different types of measurement screen targets have been presented in the literature.

Measurement screens are generally solid sheets or paper with a thickness of approximately one millimeter and low thermal conductivity. These screens are placed inside a stream of the gas to be measured and temperature distribution occurs on the surface via convective heat transfer. Because the sheets or paper used are thin, the temperature distribution detected by the infrared camera reflects the temperature distribution of the gas stream. However, as these sheets with vast surface area exchange heat via radiation with surrounding surfaces, the environmental impact should be calculated and differentiated from the obtained results. In addition, as the measurement screen acts as a barrier to the stream, it can only be applied to low-velocity flows in order to not disrupt the general nature of the stream.

The first researchers to report on using the measurement screen method were Anderson et al. (1991) and then Burch et al. (1992), who employed this method to determine the temperature distribution in the aeration streams in a car. A fiberglass sheet (plane target) with a thickness of $0.25 \mathrm{~mm}$ and a porosity of $70 \%$ was used as a measurement screen. Comparisons between the results obtained with the measurement screen and those obtained with a calibrated thermocouple showed that the temperature distribution could be determined with an accuracy of $\pm 1.3 \mathrm{~K}$. Furthermore, the time response of the measurement screen was set to 0.3 seconds. 
Cehlin et al. (2002) used a method similar to the one proposed by Anderson et al. (1991) to determine the temperature distribution over a large area at the outlet of a low-velocity diffuser used for in-room ventilation. In this method, the planar temperature distribution could be obtained using a sufficiently thin plane (plate or paper) manufactured to have a low thermal conductivity. However, as a result of the flow area being divided in two, the flow in this method is distorted and the obtained heat distribution is not representative of the actual temperature distribution of the air. Furthermore, owing to the large surface area of the measurement screen undergoing heat transfer with the environment and the internal heat transfer of the measurement screen, the heat distribution on the target is altered and may cause the results to not to match with the heat distribution of the air. For this reason, Cehlin et al. (2002) conducted a detailed analysis of the measurement uncertainty and error calculation.

Another application of the measurement screen method reported by Neto et al. (2006) was carried out to visualize the outflow of air curtain devices and to determine their temperature distribution. Unlike the work of Cehlin et al.(2002), the air velocity was increased to $8 \mathrm{~m} / \mathrm{s}$ at the exit of the air field instead of using lower flow velocities. Although the results that they obtained were useful for determining flow structure and temperature distribution, Neto et al.(2006) stated that the nonporous measurement screen had disruptive effects on the flow owing to it acting as a barrier structure and also stated that the results of the experiments were not the same as their analytical results.

In another study using a measurement screen, Elvsén and Sandberg (2009) investigated a floating jet in a ventilated room. The instantaneous velocity and temperature distributions near the diffuser were visualized using different flow visualization methods, such as particle streak velocimetry (PSV), particle image velocimetry (PIV), and infrared thermography methods. They intended to reduce the radiation to the backside by using a double-layer measurement screen, as suggested by Cehlin et al. (2002). However, the aluminum foil used as the second layer in this method may increase the heat transfer inside the measuring screen, which can cause measurement errors to increase. 
Another study in which the measurement screen was porous was carried out by Neely (2008). The study was aimed at increasing the visibility of the screen and reducing its effects on the flow by using net measurement screens with a high porosity and a high spreading coefficient. In this method, the wires forming the measurement screen must be thin enough to reach the temperature of the gas to be measured, the net of the net target must be tight enough to ensure proper data in every pixel of the camera, and the net material must have a low reflection coefficient to reduce the reflections coming from its surroundings to the minimum. For this reason, different parameters (material type, flow characteristics, placement of the measurement screen, etc.) were tried in Neely's study (2008) when measuring the flow at different temperatures to see their effects on the performance of the method. In their results, even though the measurement screen was placed perpendicular to the flow and only generated small effects on it, the background flow behind the screen was influenced by the presence of the screen and the turbulence levels of the flow increased.

Gallo et al. (2010) proposed a new measurement method based on infrared temperature measurements to determine the temperature distribution in hot gas flows. They calculated the local convection coefficient and temperature of fluids by visualizing the temperature distribution on a high porosity net of electrically conductive thin wires with an infrared camera.

The natural convection on a heated plate was investigated by Vinnichenko et al. (2016). The temperature distribution was obtained with a measurement screen that was composed of rectangular targets. The results obtained with this measurement screen were compared with the results obtained via the background-oriented schlieren method and a numeric study.

Beside these studies, another measurement screen method was developed and named "meshed infrared thermography" (MIT) by the authors of this work (Karadeniz et. al. 2013). The temperature distribution at the outlet of a split air conditioner indoor unit was visualized using this method. In this method, the temperature is measured using spheres that are placed in pre-determined intervals on nylon threads that are stretched between the two edges of a frame. This frame is placed in the flow in such a way so as to have minimum effects on it. The 
benefits of the MIT method are minimal disruption on the flow and convenient measurement of the planar heat distribution using the spheres.

Besides this method, the laser induced fluorescence (LIF) method, which can measure fluid temperature, has been used in previous research (Sakakibara and Adrian 1999, Bruchhausen et. al. 2005, Einecke et. al. 2000). In the LIF method, particles that are fluorescent when illuminated with powerful laser beams are added to the flow. After illuminating the area to be reviewed, the particles are imaged using a high-speed camera. Temperature is measured based on the fluorescence wavelength of the particles. This method is fairly expensive owing to the high cost of the components required, such as a high-speed camera and lasers. Furthermore, because the particles applied to the fluid are toxic to human health, certain precautions have to be taken where the experiment takes place. There is another whole-field temperature measurement method that utilized thermochromic liquid crystals exists in the literature (Dabiri 2009). LIF and thermochromic liquid crystals methods both have the advantage of measuring temperature and velocity at the same time.

The Rayleigh thermometry method has been reported in the literature as a method to determine air temperature (Miles and Lempert 1990). In this method, the flow is seeded with tracer particles and the stream is visualized via laser illumination. The Rayleigh method differs from the LIF method in that the wavelength of the added particles is smaller than the wavelength of the transmitted laser. Therefore, contamination of the investigated flow by dust or other particles causes measurement errors so this method can only be used in clean laboratories. Moreover, the Rayleigh method can only be used at high temperatures, and is expensive in terms of the cost of the required components, much like the LIF method. In addition, temperature can only be measured from a thin plane when using the Rayleigh method. Therefore, it is not suitable for volumetric temperature measurements.

There are also other wire based methods that can provide volumetric temperature distribution measurement. These methods can be categorized in two groups. The first group is thermistors (thermoresistive temperature sensors). In this method, the conductivity sampling was utilized for temperature field measurement (Ritterath et. al. 2011). There are several examples of this method (Schaefer et. al. 2013, Kelm et. al. 2016, Arlit et. al. 2018). As 
they are compared with the infrared imaging methods, these methods have the advantage of higher time resolution and lower flow distortion due to the size of the target. There are also optical fiber method which has the same advantages of the thermistor (Lomperski et. al. 2016).

Furthermore, another method to determine the air temperature (and the temperature of similar gasses) is to use a probe. Although this method is effective in determining the temperature at a given area, it can only be used to perform point measurements. Thus, in order to determine the heat dispersion in an area, the use of multi-point measurement devices is required. This leads to the formation of quite complicated systems. In cases in which measurements are carried out via thermocouple or probe scanning, temporal differences are observed, and a traverse system is required.

In the current study, the simultaneous volumetric temperature distribution of infraredtransparent flows (air, gases, etc.) was obtained with minimum distortion on the flow structure via the newly developed 3D meshed infrared thermography (3D MIT) method. In this method, the design of the target for volumetric temperature measurements are imaged using an infrared camera. The obtained images are processed digitally to obtain a volumetric temperature distribution. The results that we obtained using the 3D MIT method were compared with those obtained via the stereo particle image velocimetry (SPIV) method. The main advantage of 3D MIT method compared with other measurement methods is the ability to obtain temperature distributions simultaneously in three-dimensional form.

The main purpose of the study is to develop a measurement target that can visualize the volumetric temperature distribution which can be set up easily and inexpensively when the state of art level measurement methods cannot be reached. In addition, it can be said that when the error is below $1{ }^{\circ} \mathrm{C}$, it can be used as a measurement method in fundamental engineering calculations. 


\section{2- Method}

In general, measurement screen methods based on visualizing a solid target via infrared thermography are used for measuring temperature distribution in gaseous media because of the fact that gases are transparent at infrared wavelengths $(7-13 \mu \mathrm{m})$. Therefore, measurement screen methods are indirect measurement methods and the precision of such measurements is strongly based on the relation between the structures of the target and the flow. For successful measurements, the target should show a temperature distribution as similar as possible to that of air and it must create minimum distortion on the flow.

Convection is the dominant heat transfer mechanism between the target and the gas. Thus, the target must be designed to enhance this mechanism and hinder the other heat transfer mechanisms. For example, plane measurement screens have a wide surface area, which undergoes radiative heat transfers with its surrounding. These screens also undergo conductive heat transfers with themselves. Therefore, the temperature distribution obtained from a plane measurement screen is a function of convection with the gas to be measured, radiation with its surroundings, and conduction inside the screen. Therefore, the obtained temperature distribution from the plane screen does not directly represent the temperature distribution of the investigated gas. This effect was studied in this work (Section 4.2).

The distortion effects of measurement screens on the structure of the flow is another important phenomenon that leads to distortions on temperature measurements. Naturally, measurement screens that are installed in the flow area change the structure of the flow. However, the amount of deformation is critical. If the deformation is too high, it may lead to measuring an irrelevant flow structure instead of the desired one. The effects of the measurement screens on the flow were also studied in this work (Section 4.1).

In the present study, a measurement screen with the capability of handling all these effects was designed. We called our method the three dimensional meshed infrared thermography 3D MIT method. 
In the 3D MIT method, spheres are placed on the carrier ropes that are stretched between two parallel plates connected to one another with foot stands. The entire structure formed by these components is called the volumetric temperature measurement target (Figure 1).

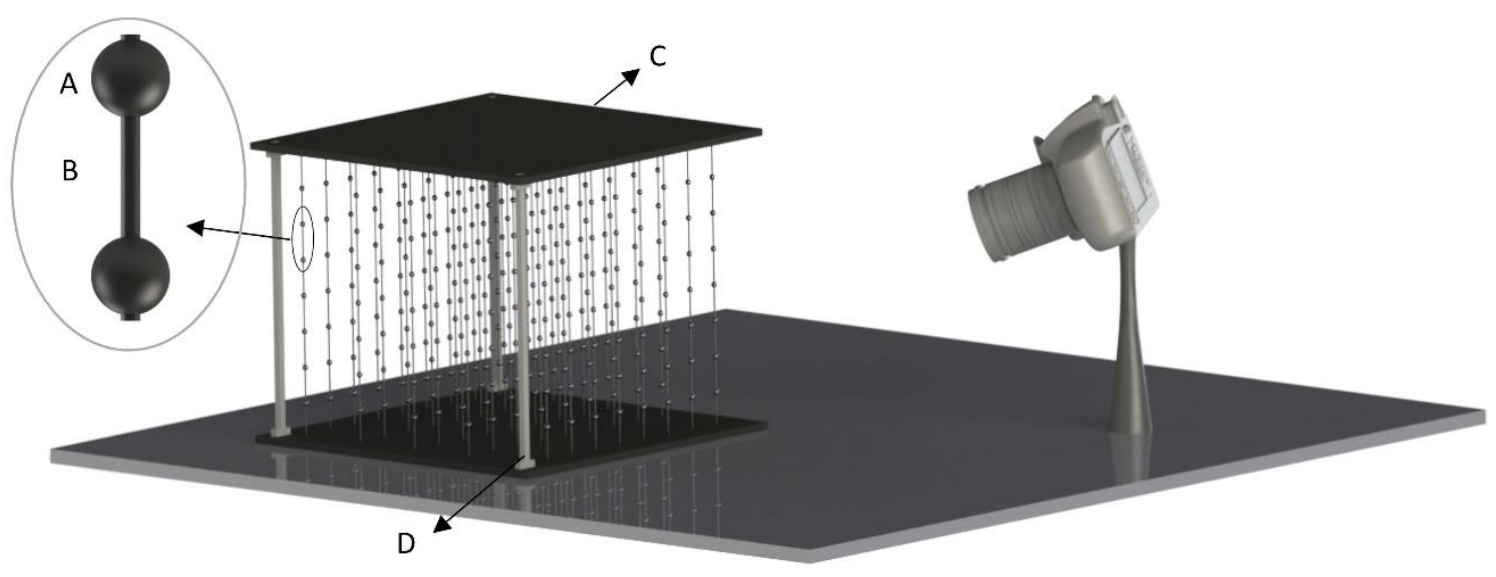

Figure 1. Three-dimensional meshed infrared thermography setup.

The spheres (Area A in Figure 1) are positioned on the carrier ropes (Area B in Figure 1), which are placed between two parallel plates (Area $\mathrm{C}$ in Figure 1). As mentioned earlier, the position of the ropes is related to the size of the spheres and the velocity of the flow. The most important parameters of the carrier ropes are their thermal conductivity and their diameter. Their thermal conductivity must be as low as possible to prevent conductive heat transfer between the spheres. Their diameter must also be small in order to reduce flow distortion. However, they must be rigid enough to hold the spheres at a fixed position. The main problem with the flickering of the spheres is the motion blur effects at the infrared image. It causes inaccurate temperature measurement. This vibration can also create extra turbulence in the flow. Making the spheres have a fixed position prevents them from flickering while they are exposed to the stream and also ensures that the image can be taken clearly.

Additionally, a single camera can capture a two-dimensional image which does not allow three-dimensional data. Therefore, in general, two cameras are used to obtain 3D data. In the presented method, the sphere's locations are fixed and known, the measurement mesh locations in three-dimensional space are also known. Thus, the image obtained from the 
thermal camera is only used for matching and temperature measurements, and 3D measurements can be performed with a single camera.

The plates (Area $C$ in Figure 1) and the supporting beams (Area D in Figure 1) are the mechanical carriers of the spheres and the ropes. They are the skeleton of the system. Their positioning is important in order to avoid disturbing the flow and visually blocking the camera.

The most important parameter of the system is the geometrical size and location of spheres. The spheres' geometry has the advantage of being symmetric. The area projected perpendicular to the free stream velocity parameter $\left(A_{f}\right)$ in the drag coefficient formula (Equation 1) (Incropera and DeWitt 1996) does not change for different attack angles. This also means that the flow deformation for every attack angle will not be affected by the target's geometry. The proposed symmetric geometry also has advantages for thermal imaging. Spheres have same view area from all view angles. Therefore, spheres are more suitable than planes and porous targets for three-dimensional flow measurements and volumetric measurements.

$$
C_{d}=\frac{F_{d}}{A_{f}\left(\rho V^{2} / 2\right)}
$$

The diameter of the spheres is a critical parameter for 3D MIT. As the size of each sphere increases, the flow deformation also increases. This also affects the location of the subsequent sphere. In ideal applications, it is expected that the flow deformation caused by a sphere will damp out before reaching the other spheres. Thus, if the flow deformation increases because the diameter of the spheres increases or because of an increase in flow velocity, the spheres must be positioned more sparsely. By the same token, decreasing the diameter of the spheres has advantageous flow effects. However, it makes temperature measurements harder. In Figure 2, this phenomenon is shown. 

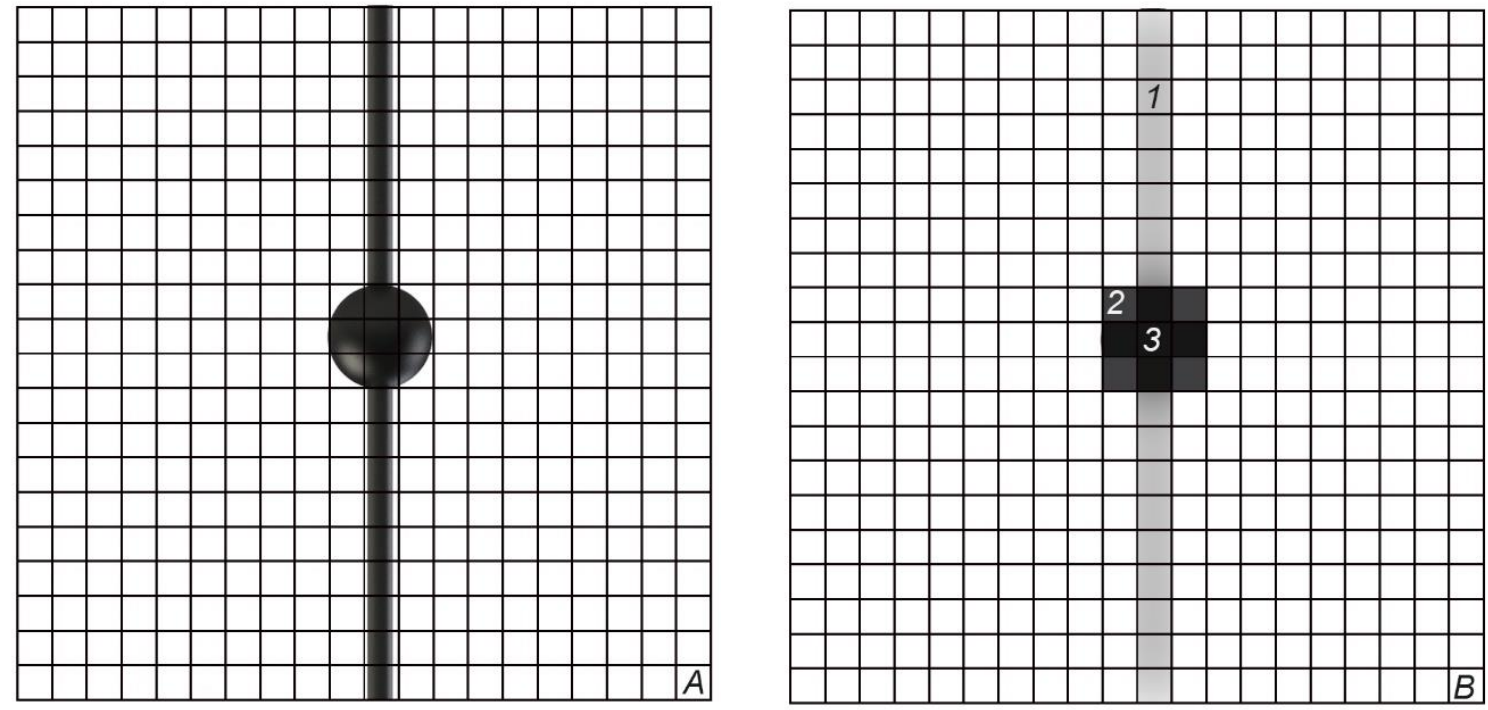

Figure 2. A) Target projection on the sensor and B) simulated temperature map.

In digital imaging, the light-sensitive area is divided into sensor areas that are called pixels (picture element). These sensors capture the light that focuses on them. Then the collected data are evaluated and converted to an image. At this digital image, every sensor (pixel) represent as a color value also named as pixel. Just like normal digital images, infrared images consist of pixels. The difference is that infrared image pixels represent temperature values instead of light intensity or color data. Figure 2-A shows the projection of an image on the sensor and Figure 2-B shows its simulated temperature map. For a precise temperature measurement, a pixel must be filled by the target surface as shown in Pixel-3 in Figure 2-B. Pixel-3 only gathers data from the target surface. Otherwise, the pixel would measure an areaweighted average of the target and the background. Pixel-1 and Pixel-2 in Figure 2-B can be examples of this phenomenon. To obtain the target temperature, subpixel algorithms must be used in these situations.

Subpixel algorithms are useful and approved methods for many applications such as remapping an image. It is also used to increase the accuracy of PIV experiments. In this sense, it can be also used for increasing the accuracy of 3DMIT or High Porosity Mesh methods. However the background temperature also must be known for efficient application. It must be noted that background may have different surface emissivity or eccentric temperature distribution. In these situation the application of this method became unreliably complex. 
The number of pixels needed for direct measure can be calculated. Let $\mathrm{n}$ be the minimum number of pixels in the related axis that should be covered by the measurement sphere in the image. The number $\mathrm{n}$ must be chosen so that at least one pixel in the image is filled by the sphere. It is advisable that this number be 3 or greater, considering that, in a real thermal image, not all spheres are concentric with pixels. Hirasawa et al. (2015) suggested that the measurement spot should be imaged by at least $6 \times 6$ pixels area. This creates a relationship between the sphere's size, the resolution of the image, the sensor's size, and the focal length of the lens. The geometrical relationships of these parameters is shown in Figure 3, and the mathematical relationships of these parameters are given in Equations 2, 3 and 4.

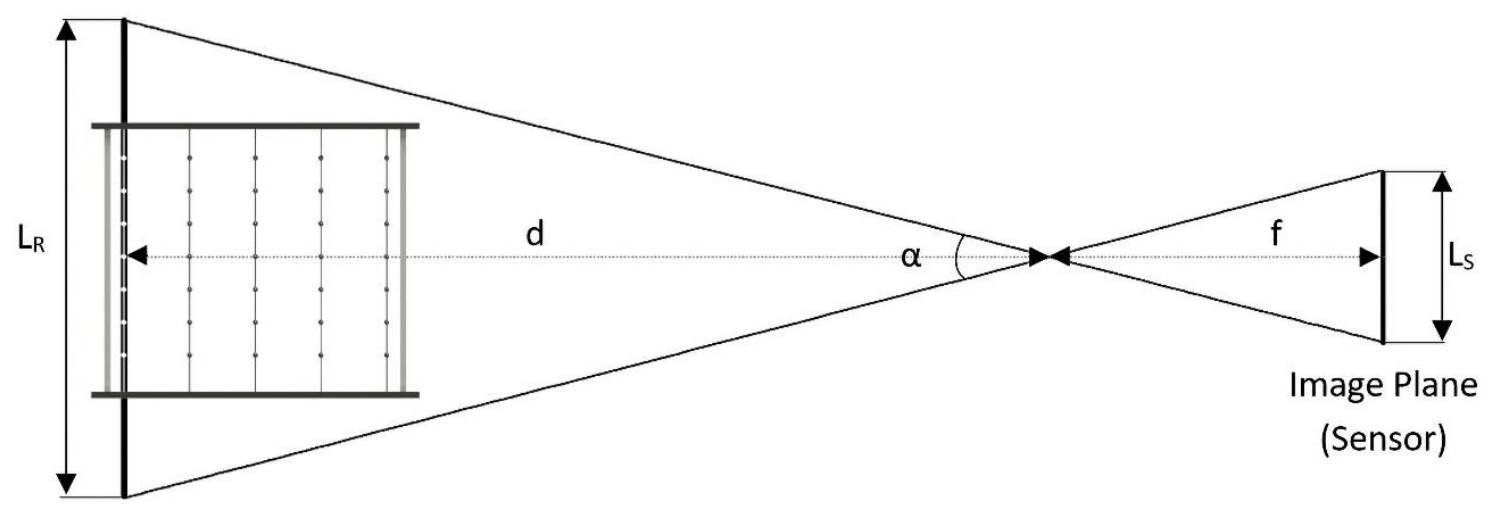

Object Plane

Figure 3. Optical schematic of the proposed 3DMIT setup.

$$
\begin{aligned}
& \frac{L_{R}}{d}=\frac{L_{S}}{f} \\
& S_{f}=\frac{L_{R}}{P \#}=\frac{D_{\text {sphere }}}{P \#_{\text {sphere }}} \\
& d=\frac{D_{\text {sphere }} P \#}{P \#_{\text {sphere }} L_{S}} f \quad\left(P \#_{\text {sphere }} \geq n\right)
\end{aligned}
$$

The area of a pixel corresponding to the object plane is defined as the scale factor $\left(S_{f}\right)$, which depends on the focal length of the lens $(f)$, the size of the sensor $\left(L_{s}\right)$, the pixel number of the 
camera at the related axis $(P \#)$, and the distance between the camera and the object plane (d). The focal length of the lens and the size of the sensor also determine the angle of view $(\mathrm{AOV})(\alpha)$ of the lens.

These equations can be used for determining the size of the analyzed volume and the size of the investigation spheres according to the specifications of the camera and the lens. However, the distance between the camera and the object plane is also related to the depth of field.

Lenses focus light that comes from a certain distance owing to their operating principle. This distance is called the focusing distance (s), and objects at these distances are imaged with the maximum sharpness allowed by the lens. As objects move away from or closer to the lens from this distance, the area scattered in the image plane by light coming from a single point expands. The diameter of this scattered area which cannot be visually observed and does not negatively affect the technical process is called the permissible diameter of the circle of confusion (COC). This phenomenon is also explained by Minkina and Dudzik (2009). In short, the region that is close enough to the focusing distance so that the diameter of the scattered area of the light coming from a point source is smaller than the $\mathrm{COC}$ is considered as focused; this region is called the depth of field. Formulas related to the depth of field are given in Equations 5, 6, 7 and 8 (Jacobson 1978). Hyper-focal (H) is the closest distance that rays from a point can reach the lens parallel only. It is also used for calculation of depth of field (DOF). The near focus distance $\left(D_{N}\right)$ is the closest object distance that can be focused on the image. In the same way, the far focus distance $\left(D_{F}\right)$ is the farthest distance that can be focused on the image. DOF is the total distance between $D_{F}$ and $D_{N}$.

$$
\begin{aligned}
& H=\frac{f^{2}}{N \cdot c}+f \\
& D_{N}=\frac{H \cdot s}{H+s} \\
& D_{F}=\frac{H \cdot s}{H-s} \\
& D O F=\frac{2 H \cdot s^{2}}{H^{2}-s^{2}}
\end{aligned}
$$


Lack of focusing (blurriness) in terms of the thermal imaging means that temperature information from different points intermingle and cause the measured temperature to be incorrect (It causes the data of the pixels to blend with each other). Therefore, defocusing cannot have a fixed amount of error. The temperature measurement error is directly related to the temperature differences of the measured areas. As the temperature difference of the blended pixels increases, the temperature error caused by the defocusing also increase. On the other hand, if a constant temperature target with no temperature gradient was imaged, defocusing would not cause any measurement error. As a daily life example of this phenomena, it can be said that the focused and the unfocused image of a flat blue sky would give the same result. Thus, the $\mathrm{COC}$ should be as small as possible according to the pixel size of the sensor in order to minimize this blurriness.

The aperture number $(\mathrm{N})$ and the focal length (f) parameters are not configurable in most thermal cameras. Therefore, the focusing distance $(s)$ is the main adjustable parameter for arranging the depth of field in which the 3D MIT target must fit. Because of this, it is necessary to check whether the distance between the lens and the object plane (d) obtained from Equation 4 is compatible with the calculation of DOF in Equation 8.

The final result of the aforementioned implications is that the spheres should be small enough to minimize flow distortions and large enough to be captured by the infrared camera. If the infrared camera technology enables higher resolution, the spheres can be smaller. In some situation even the wires itself can be used for measurement. However right now conventional infrared cameras' resolution not enough achieving direct measurement from wires.

The location and the size of the spheres has to be selected to satisfy both requirements. The maximum number of the spheres used in this study is 315 . However, this number is not a global maximum. This number may vary according to the area to be investigated, the thermal camera and the lens. The dimensions of the spheres can be obtained using the equations given. The distance between the spheres is related to the structure of the flow and the size of the spheres. The dimensions of the experiment setup that is used in the study is given in Table 1. 
Table 1. Dimensions and specifications of the 3D MIT target that is used in the current study

\begin{tabular}{|c|c|c|}
\hline $\begin{array}{l}\text { Components of the } \\
\text { 3D MIT target }\end{array}$ & Dimensions / Specifications & Notes \\
\hline Target & $210 \mathrm{~mm} \times 250 \mathrm{~mm} \times 250 \mathrm{~mm}$ & $\begin{array}{l}\text { The target was designed to cover } \\
\text { the flow area of the investigated } \\
\text { fan. }\end{array}$ \\
\hline Parallel plates & $\begin{array}{c}5 \mathrm{~mm} \times 250 \mathrm{~mm} \times 250 \mathrm{~mm} \\
\text { Quantity: } 2\end{array}$ & $\begin{array}{l}\text { They were placed out of the flow in } \\
\text { order to avoid disturbing the flow } \\
\text { and visually blocking the camera. }\end{array}$ \\
\hline Carrier ropes & $\begin{array}{c}1 \text { mm diameter, } \\
\text { Quantity: } 45 \\
\text { Materials: Nylon Thread } \\
\text { Thermal conductivity }=0.31 \\
\text { W/mK [12] }\end{array}$ & $\begin{array}{l}\text { Their thermal conductivity must be } \\
\text { as low as possible to prevent } \\
\text { conductive heat transfer between } \\
\text { the spheres. Their diameter must } \\
\text { also be small in order to reduce } \\
\text { flow distortion. However, they } \\
\text { must be rigid enough to hold the } \\
\text { spheres at a fixed position. }\end{array}$ \\
\hline Supporting beam & $\begin{array}{c}210 \mathrm{~mm} \times 5 \mathrm{~mm} \times 5 \mathrm{~mm} \\
\text { Quantity: } 3 \\
\text { Thermal conductivity }=0.16 \\
\mathrm{~W} / \mathrm{mK}\end{array}$ & $\begin{array}{c}\text { They were placed out of the flow in } \\
\text { order to avoid disturbing the flow } \\
\text { and visually blocking the camera. } \\
\text { They were made of ABS using 3D } \\
\text { printer. }\end{array}$ \\
\hline Sphere & $\begin{array}{c}4 \mathrm{~mm} \text { diameter } \\
\text { Thermal conductivity }=401 \\
\mathrm{~W} / \mathrm{mK}[12]\end{array}$ & $\begin{array}{l}\text { The sphere's diameter is chosen } \\
\text { small enough to minimize flow } \\
\text { distortions and large enough to be } \\
\text { captured by the infrared camera. } \\
\text { Their material was copper. }\end{array}$ \\
\hline $\begin{array}{l}\text { Total number of } \\
\text { spheres }\end{array}$ & Quantity: 315 & $\begin{array}{l}\text { It should be enough pieces of the } \\
\text { sphere to measure the entire flow } \\
\text { field. }\end{array}$ \\
\hline $\begin{array}{l}\text { Distance between } \\
\text { two spheres }\end{array}$ & $25 \mathrm{~mm}$ & $\begin{array}{l}\text { It is expected that the flow } \\
\text { deformation caused by a sphere } \\
\text { will damp out before reaching the } \\
\text { other spheres. }\end{array}$ \\
\hline
\end{tabular}

An infrared camera (Flir T400) that has a resolution of $320 \times 240$ pixels was used in this study. The camera has an accuracy of $\pm 50 \mathrm{mK}$ at $30{ }^{\circ} \mathrm{C}$ and it is sensitive to infrared radiation in a wavelength range of 7-13.5 $\mu \mathrm{m}$. 


\subsection{Thermal inertia of the 3D MIT spheres}

If a non-steady state flow will be investigated, the thermal inertia is critical for all the measurement screen methods. As the mass of the measurement screen and the materials' thermal capacity of the measurement screen increases the thermal inertia effect also increases. Among the measurement screens that were used in this study, 3D MIT measurement screen has a lower thermal inertia effect due to its smaller mass. The time response of a sphere can be calculated by the equations 9-13.

$$
\begin{aligned}
& \operatorname{Re}_{D}=\frac{V D}{v} \\
& \overline{N u_{D}}=2+\left(0.4 \operatorname{Re}_{D}^{1 / 2}+0.06 \operatorname{Re}_{D}^{2 / 3}\right) \operatorname{Pr}^{0.4}\left(\frac{\mu}{\mu_{s}}\right)^{1 / 4} \\
& \bar{h}=\overline{N u}_{D} \frac{k}{D} \\
& B i=\frac{\left.-r_{0} / 3\right)}{k_{s}} \\
& t=\frac{\rho c_{p} D}{\sigma_{h}} \ln \frac{T_{i}-T_{\infty}}{T-T_{\infty}}
\end{aligned}
$$

As the equations 9-11 were followed, Reynolds number $\left(\operatorname{Re}_{\mathrm{D}}\right)$ calculated as 2727.24 , the average Nusselt number $\left(\overline{\boldsymbol{N u}_{\boldsymbol{D}}}\right)$ calculated as 30.34 , the average convection coefficient $(\overline{\boldsymbol{h}})$ calculated as $209.03 \mathrm{~W} / \mathrm{m} 2 \mathrm{~K}$. For equation 13 to be valid, Biot number ( $\mathrm{Bi}$ ) also must be evaluated. Bi calculated as $3.75 \times 10^{-4}$ by using equation 12 . So equation 13 can be used because of $\mathrm{Bi}<<1$. The time required for a $0.5 \mathrm{~K}$ heating of a sphere is 7.64 seconds at $1 \mathrm{~K}$ temperature difference. It must be noted that the spheres have cylindrical holes that carrier ropes pass through. A small amount of strong adhesive used in this hole for fixing the position of the spheres. These holes were neglected in the calculations. These data show that current time response of the system does not allow investigation of non-steady-state flow fields. Reducing 
size of the spheres decrease the response time (Figure 4). Using spheres smaller than $2 \mathrm{~mm}$ will reduce the response time to less than $1 \mathrm{~s}$. Also, the reduction in sphere size is an advantage in terms of flow effects. However, for accurate measurement, camera selection and distance adjustment must be done according to the equations given in the previous section. In this study, $4 \mathrm{~mm}$ copper spheres were selected due to their advantage of visibility. Copper was selected as the sphere material for its high thermal diffusivity which shortens the response time of the spheres. High thermal diffusivity also prevents forming of a temperature gradient on the sphere so thermal imaging can be done with any angle.

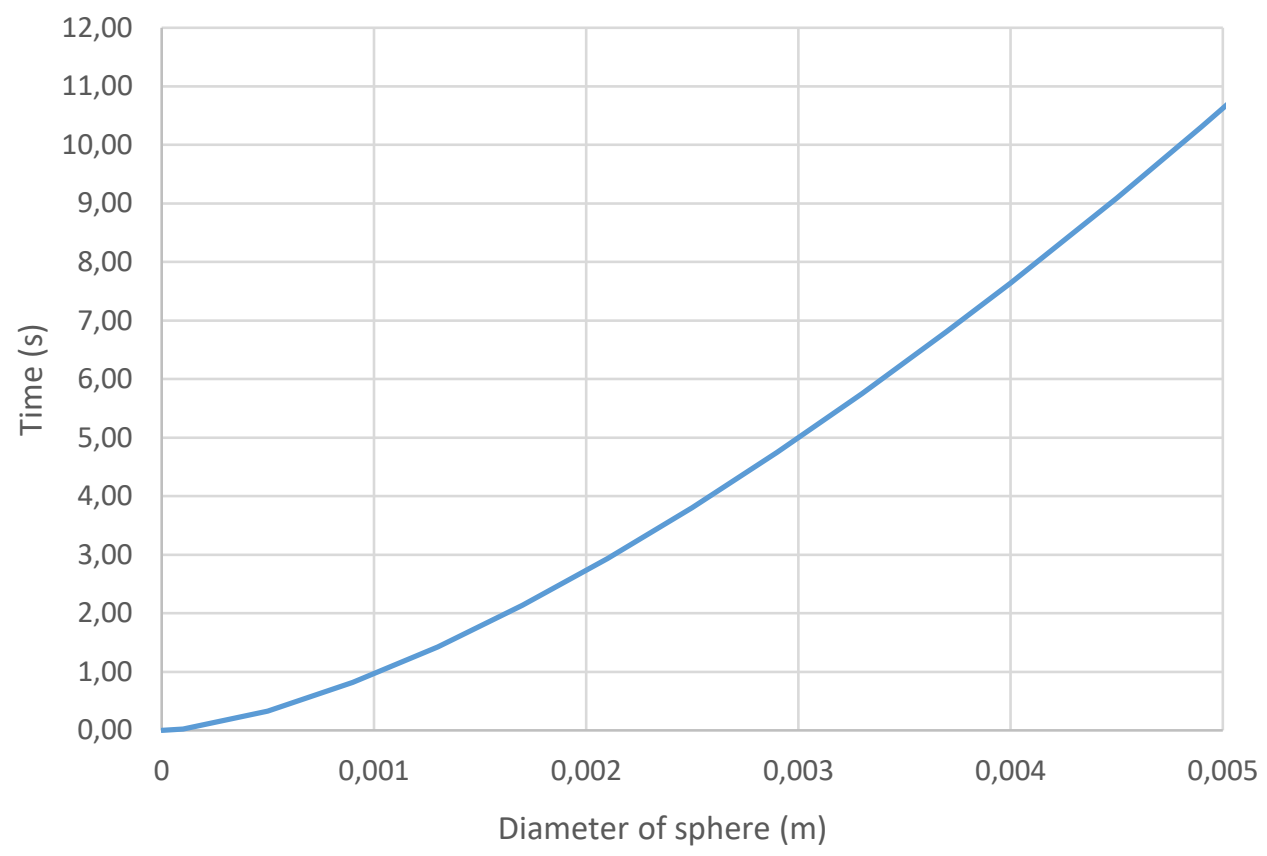

Figure 4. The effect of sphere diameters on the time required for a $0.5 \mathrm{~K}$ heating of a sphere at $1 \mathrm{~K}$ temperature difference.

\section{3- Validation}

A comparative experiment was carried out using thermocouples to validate the temperatures obtained via the 3D MIT method. In this experiment, the 3D MIT target was placed at the exit of a cross-flow fan (CFF) that drives hot air. The experimental setups are shown in Figure 5. 


\section{The Infrared Camera}

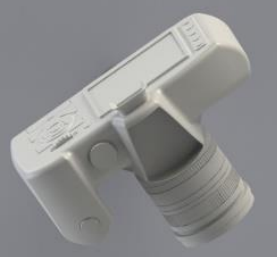

A

3D MIT Target

\section{Hot Air}

Source

Hot Air

The Infrared Camera

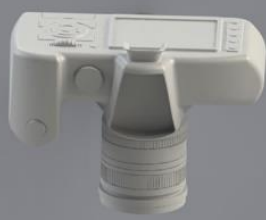

Hot Air

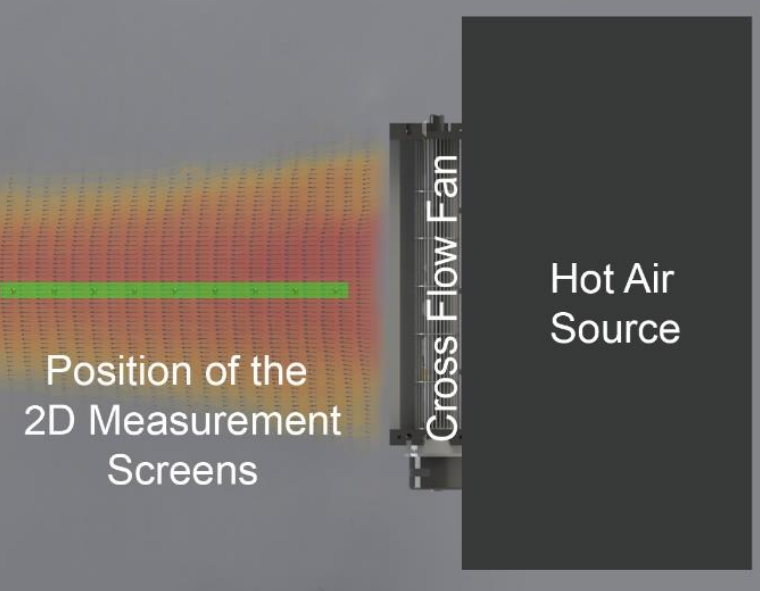

$\mathrm{B}$

Figure 5. The schematic of the experimental setups. A) 3D MIT experimental setup B) 2D measurement screens experimental setup 
The dimensions of the exit of the CFF were $180 \times 40 \mathrm{~mm}^{2}$. Reynolds number of a cross flow fan can be calculated according to Equation 14 and 15 (Lazzarotto et al. 2001). ( (n) rotational speed of fan (rpm), (D) impeller external diameter $(\mathrm{mm}),\left(\mathrm{c}_{b}\right)$ blade chord $(\mathrm{mm})$ and $(v)$ kinematic viscosity $\left.\left(\mathrm{m}^{2} / \mathrm{s}\right)\right)$ The Reynolds number of the flow was calculated as 4880 .

$$
\begin{aligned}
& \operatorname{Re}_{e}=\frac{u_{L} c_{p}}{v} \\
& u_{L}=\frac{2 \pi n}{60} \cdot \frac{D_{2}}{2}
\end{aligned}
$$

K-type thermocouples were used in the current study. They were tested by using a constant temperature water-bath before the validation study. It was observed that the absolute difference from the bath temperature was between $0.1 \mathrm{~K}$ and $0.5 \mathrm{~K}$, whereas the standard deviation of the measured temperatures from the different thermocouples was $0.15 \mathrm{~K}$.

Thermocouples were placed beside five of the measurement spheres. These validation points were positioned on a straight line that went through the center of the fan exit area perpendicularly. The distance between the validation points was $25 \mathrm{~mm}$. Temperature measurements were performed with thermocouples and via the 3D MIT method simultaneously. The validation experiments repeated 5 times. The linear regression fitting results between the 3D MIT and the thermocouple measurements are shown in Figure 6. 


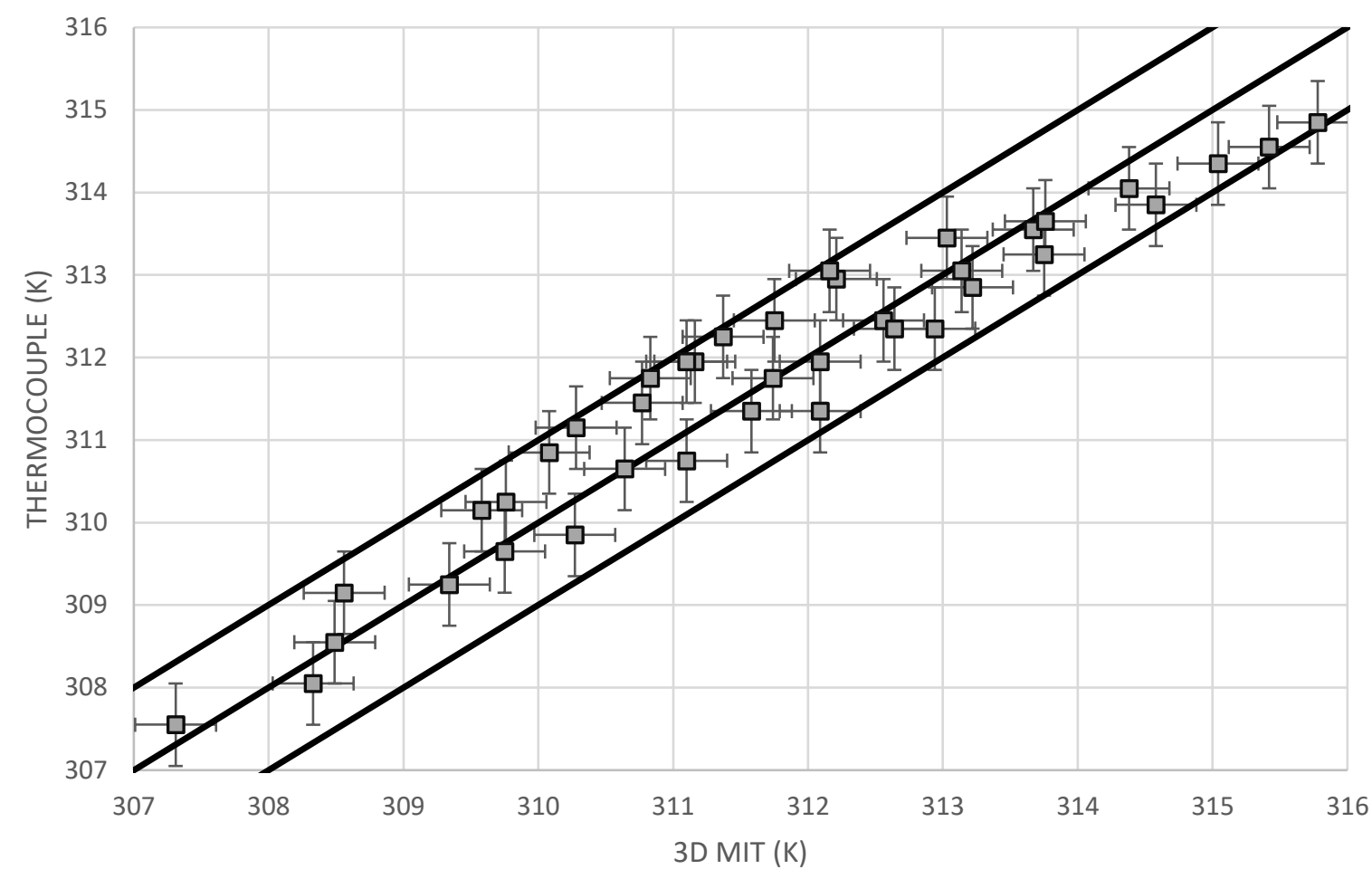

Figure 6. Linear regression fitting between the measurements of both validation experiments.

After examining all the data presented in Figure 6, we determined that the maximum error was less than $1 \mathrm{~K}$. The absolute difference values between the thermocouple and the 3D MIT measurements varied from 0.01 to $0.93 \mathrm{~K}$. The average difference between the two experiments was $0.06 \mathrm{~K}$. The standard deviation of the absolute differences for all the measurement nodes was $0.3 \mathrm{~K}$.

After the results were analyzed, we determined that the 3D MIT results are in good agreement with the thermocouple measurements. This shows that the 3D MIT method is useful for both measuring and visualizing temperature distribution. A comparison of this method with alternative methods is given in Section 5, and the advantages of using this measurement method are presented again. 


\section{4- Experimental comparison of two-dimensional targets}

For this part of our study, the meshed infrared thermography method was used with other measurement screens (the plane measurement screen and the high porosity measurement screen) for experimental comparisons.

A metal frame with a thickness of $1 \mathrm{~mm}$ was used for holding all the measurements screens. The plane measurement screen was made of a paper-based material with a thickness of approximately $0.1 \mathrm{~mm}$. The high porosity measurement screen was made of a plastic-based material with a radius of approximately $0.2 \mathrm{~mm}$. The gap between the wires was approximately $1.4 \mathrm{~mm}$. A two dimensional MIT target was also built for fair comparison. The target has same parameters (Sphere and Carrier rope properties) with the validated 3D target. All targets were painted with the same black paint (emissivity: 0.98). IR measurements cross checked with thermocouples without jet flow applied.

The three measurement screens (the plane, high porosity and MIT measurement screens) were investigated in terms of temperature measurement performance and flow distortion. The same CFF that was used for the validation experiments was also used in these comparative experiments. It should be noted that these comparisons were made in two dimensions due to the fact that plane and high porosity measurement screens cannot be applied in threedimensional measurements.

\subsection{Investigation of the effects of measurement screens on flow structures via particle image velocimetry (PIV)}

PIV is a flow visualization method that can be used for both qualitative and quantitative measurements. It was discovered in the early 1980s (Adrian 2015). In this method, the flow is seeded with tracer particles that can move coherently with the flow and reflect light. When these particles cross the area of interest, they are illuminated with a high-power light source that creates a light sheet for imaging with PIV cameras. The details of the technique can be studied by the reference book written by Adrian and Westerweel (2011) and Raffel et al (2007). 
The PIV method was used to clearly examine the effects of the measurement screens on the flow.

\subsubsection{Particle image velocimetry setup}

In this study, a $15-\mathrm{Hz}, 135-\mathrm{mJ}$ double pulse ND:YAG laser was used to illuminate the particles. Two 4-MP cameras (FlowSense MKII) and $50 \mathrm{~mm}$ lenses (Zeiss $50 \mathrm{~mm}$ f2 macro planar) were used for the SPIV setup. The time between pulses for image pairs was selected to be $40 \mu \mathrm{s}$. A two-layer calibration target used for SPIV calibration. For each experiment, 400 images were taken using two cameras to generate 200 two-dimensional, three-component vector maps.

In the data processing stage, the DynamicStudio software (version 3.41.38) was used with all the experiment setups to apply the average correlation and adaptive correlation methods. The interrogation areas were selected as $32 \times 32$ pixel regions with $50 \%$ overlap. Then, using the vector maps obtained from both cameras and the calibration data, the SPIV results were obtained (Özer and Kumlutaş 2017).

\subsubsection{Results of the particle image velocimetry experiments}

In order to clearly investigate the flow distortion effects, PIV data was gathered from the top view of the measurement screens. First, the measurement screens were placed parallel to the main flow direction at the core of the jet. The PIV measurement results are presented in Figure 7. 


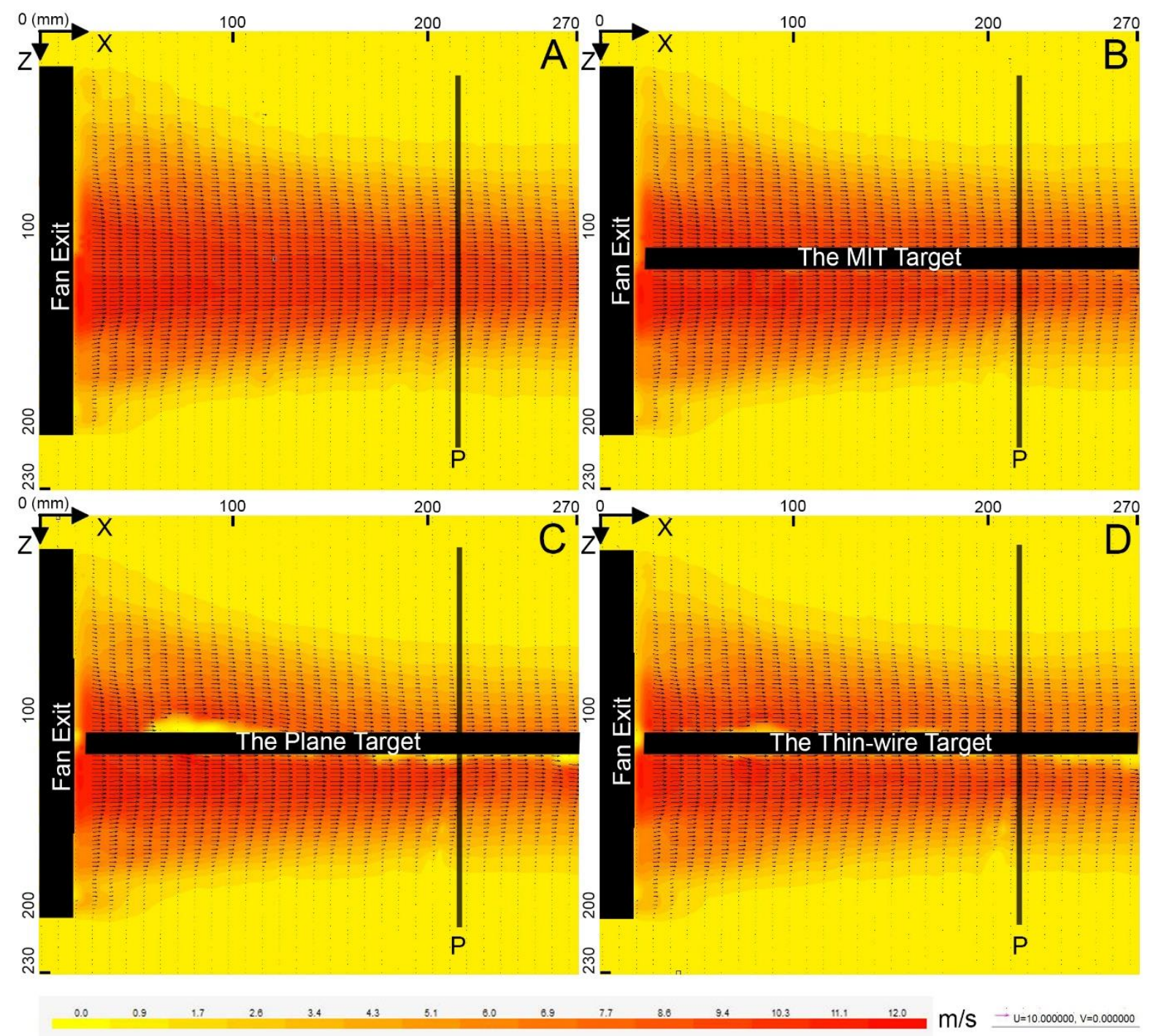

Figure 7. PIV comparison of the measurement screens, which were placed parallel to the jet flow. a) Results without a measurement screen, and results using b) the MIT target, c) the plane target, and d) the high porosity target.

After analyzing the results shown in Figure 7, we found that the MIT target caused the least distortion in the flow profile. However, it should be noted that, in general, placing the targets parallel to the flow does not cause a significant deformation in the flow profile. The position of the flow profile plane shown in Figure 7 and 9. The obtained profiles are presented in Figure 8. 


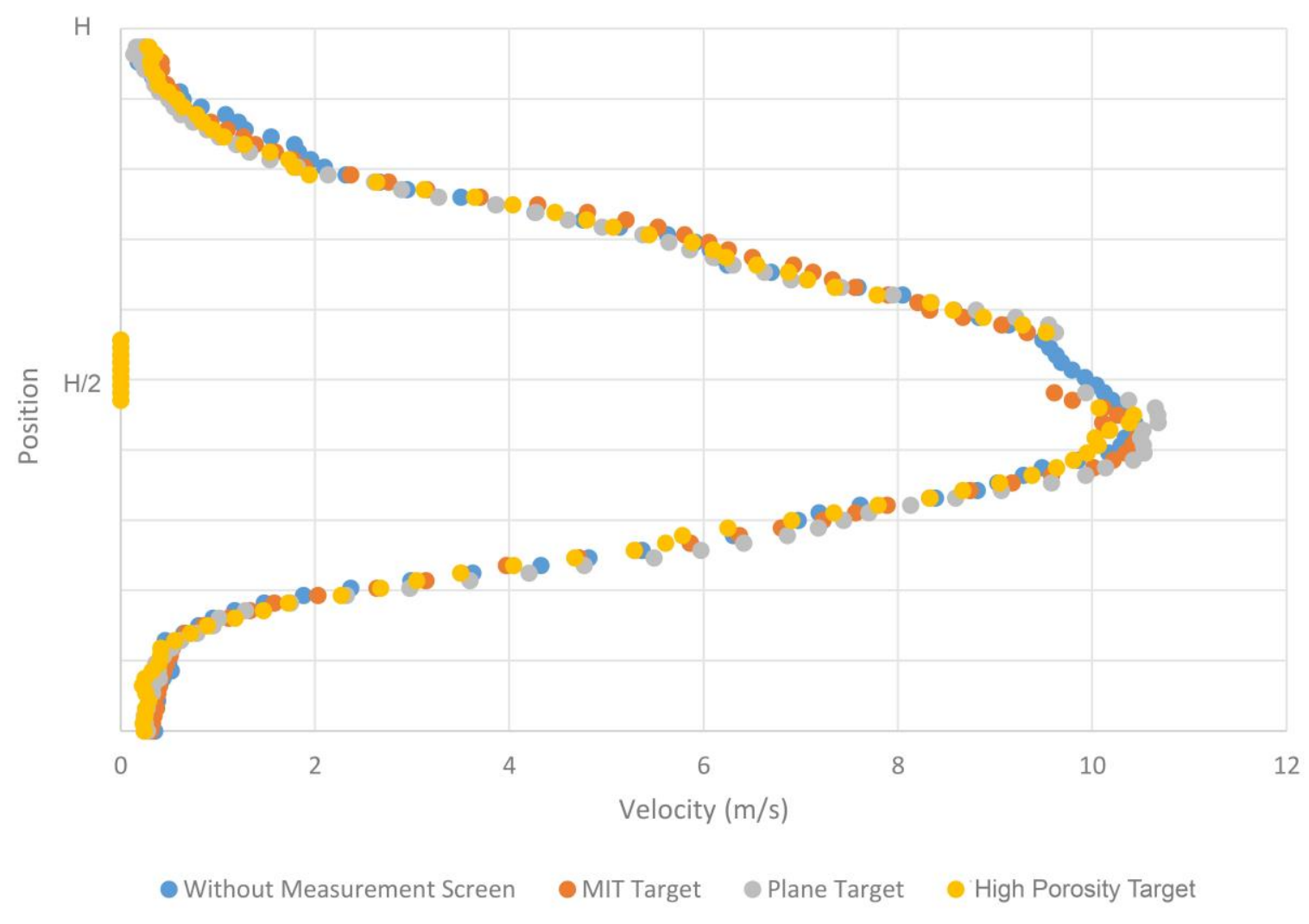

Figure 8. Comparison of different measurement screens' flow profiles obtained by PIV (The measurement screens are placed parallel to the jet flow.)

In Figure 8, the gap in the flow profiles corresponds to the measurement screen placement. The gap wider than the spatial obstruction of the various frames, ropes or planes because of the reflections caused by the PIV laser. Surface reflection prevented getting data from there. The asymmetry of the flow profiles was caused by shaft gap of the CFF. Therefore, the maximum velocity does not occur at the middle plane of the exit [Eck 1973].

It can be seen that the obtained profiles are not significantly different. The main reason for this, in the case of the measurement screens placed parallel to the flow, is that the difference between the areas projected perpendicular to the free flow velocity of the measurement screens is small. The absolute average differences between the flows with and without measurement screens were calculated by comparing the flow profiles shown Figure 8 . The velocity drop percentages caused by the MIT target, the plane target, and the high porosity target were $3.36 \%, 4.79 \%$, and $3.40 \%$. 
The uncertainty of the PIV data evaluated by the means of image mapping, interrogation area, resolution, image sampling. It is estimated about $1 \%$. A repeatability experiment was done. The difference between the two experiments was $0.89 \%$.

As the uncertainty of the measurement taken into the account, it can be said that the MIT target and the high porosity target create a similar amount of distortion to the flow. According to these results, the plane target creates a slightly higher flow distortion among the measurement screens used owing to its geometry, which splits the flow.

Our results show that the measurement screens do not create too much distortion in the flow in cases where they are placed parallel to the flow structure. However, it may be desirable to use the proposed measurement method in cases where the direction of the flow structure to be examined is unknown. In order to examine these situations, a second experiment was carried out. In this experiment, the target was placed at an angle of 30 degrees with the flow. The PIV measurement results are presented in Figure 9. 


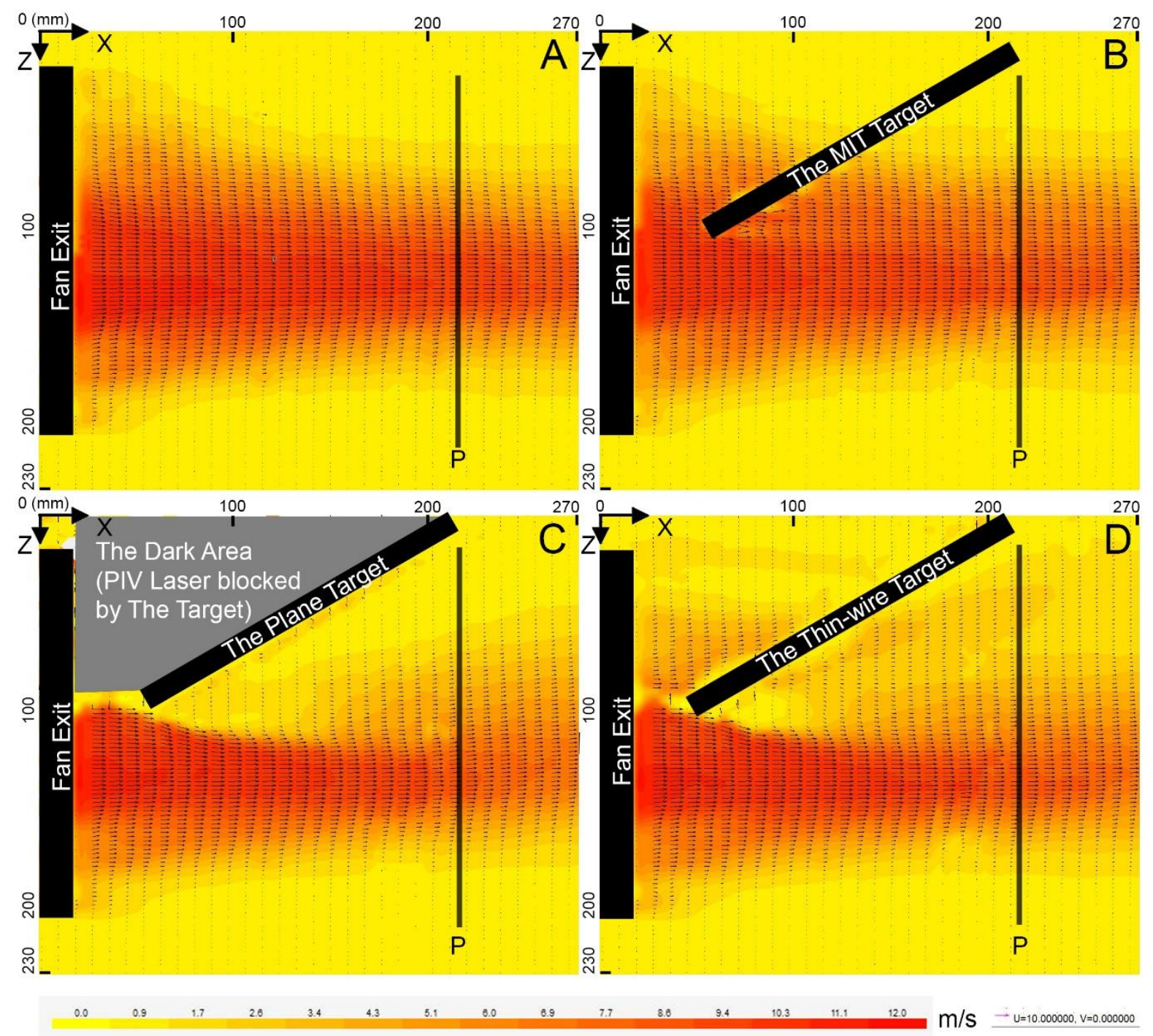

Figure 9. PIV comparison for measurement screens placed at an angle of 30 degrees with the flow. a) Results without a measurement screen, and results b) with the MIT target, c) the plane target, and d) the high porosity target.

By examining Figure 9, the measurement screen effects on the flow can be more clearly observed because of the changes of the areas projected perpendicular to the free flow velocity of the measurement screens. The absolute average differences between the flows with and without the measurement screens were calculated by comparing the flow profiles shown in Figure 10 . The velocity drop percentage caused by the MIT target, the plane target, and the high porosity target were $3.68 \%, 24.51 \%$, and $14.33 \%$, respectively. By evaluating these results, it can be clearly observed that the MIT target caused the least distortion effects on the flow. Another important point to make is that the flow distortion of the MIT target does 
not vary according to its position in the flow. This is an important advantage both for studying unknown flows and for investigating three-dimensional temperature distributions. On the other hand, the plane target's flow distortion effects increased from $4.79 \%$ to $24.51 \%$, while those for the high porosity target increased from $3.40 \%$ to $14.33 \%$.

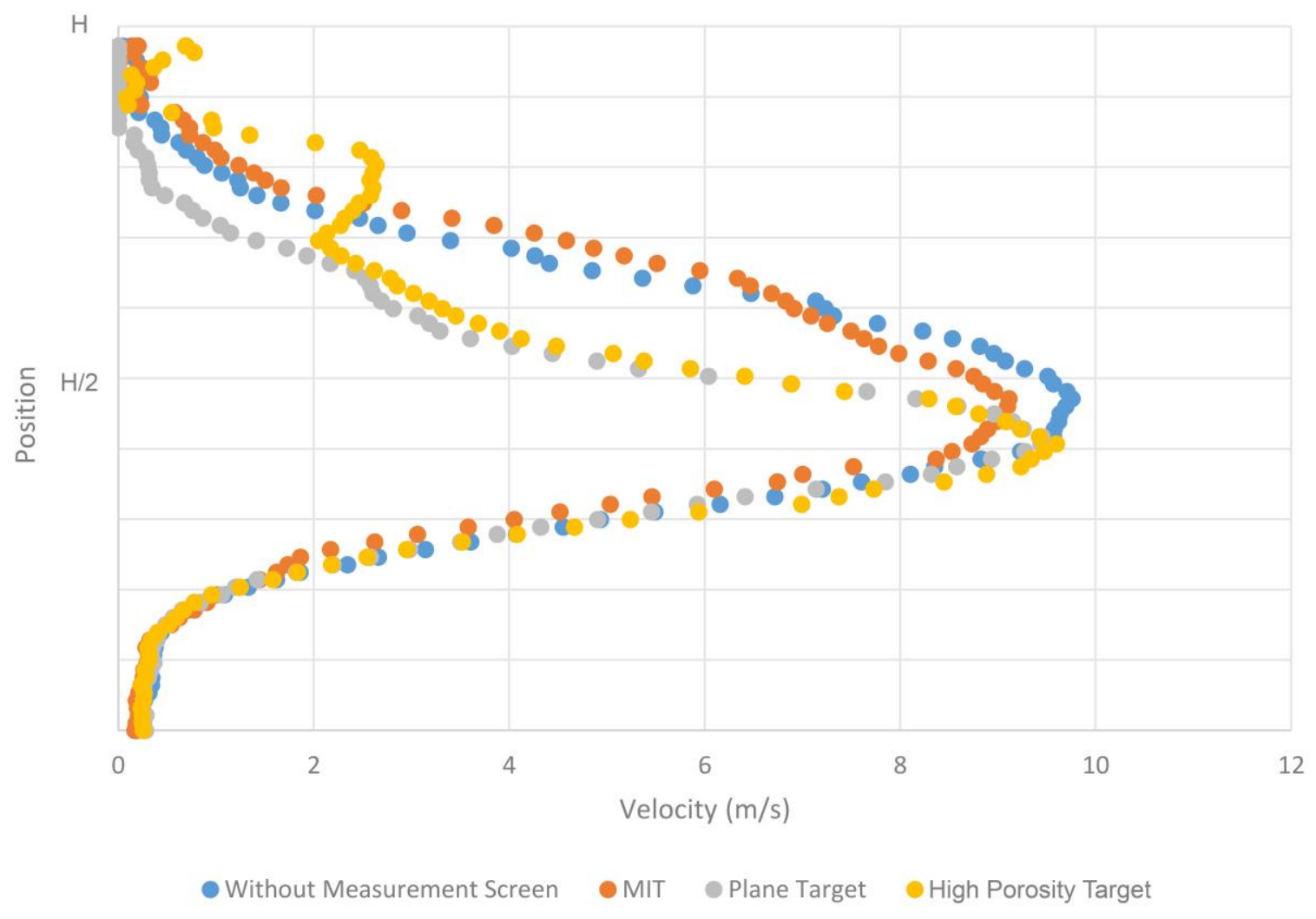

Figure 10. Comparison of different measurement screens' flow profiles obtained by PIV (The measurement screens are placed at an angle of 30 degrees with the flow)

\subsection{Comparison of the temperature measurement capabilities of the measurement screens}

The experimental setup used for validating the MIT method was also used for a comparison of the temperature measurement capabilities of different measurement screens. Five measurement points were selected in the core of the jet for a quantitative comparison. The gap between two points was $25 \mathrm{~mm}$. Temperature measurements with thermocouples were also carried out at these points to validate the comparison. 
In Figure 11, the temperature values measured at the aforementioned points are shown. Figure 12 shows the temperature distributions obtained with the MIT target, the plane target, and the high porosity target. The temperature measurement points are also marked in Figure 12.

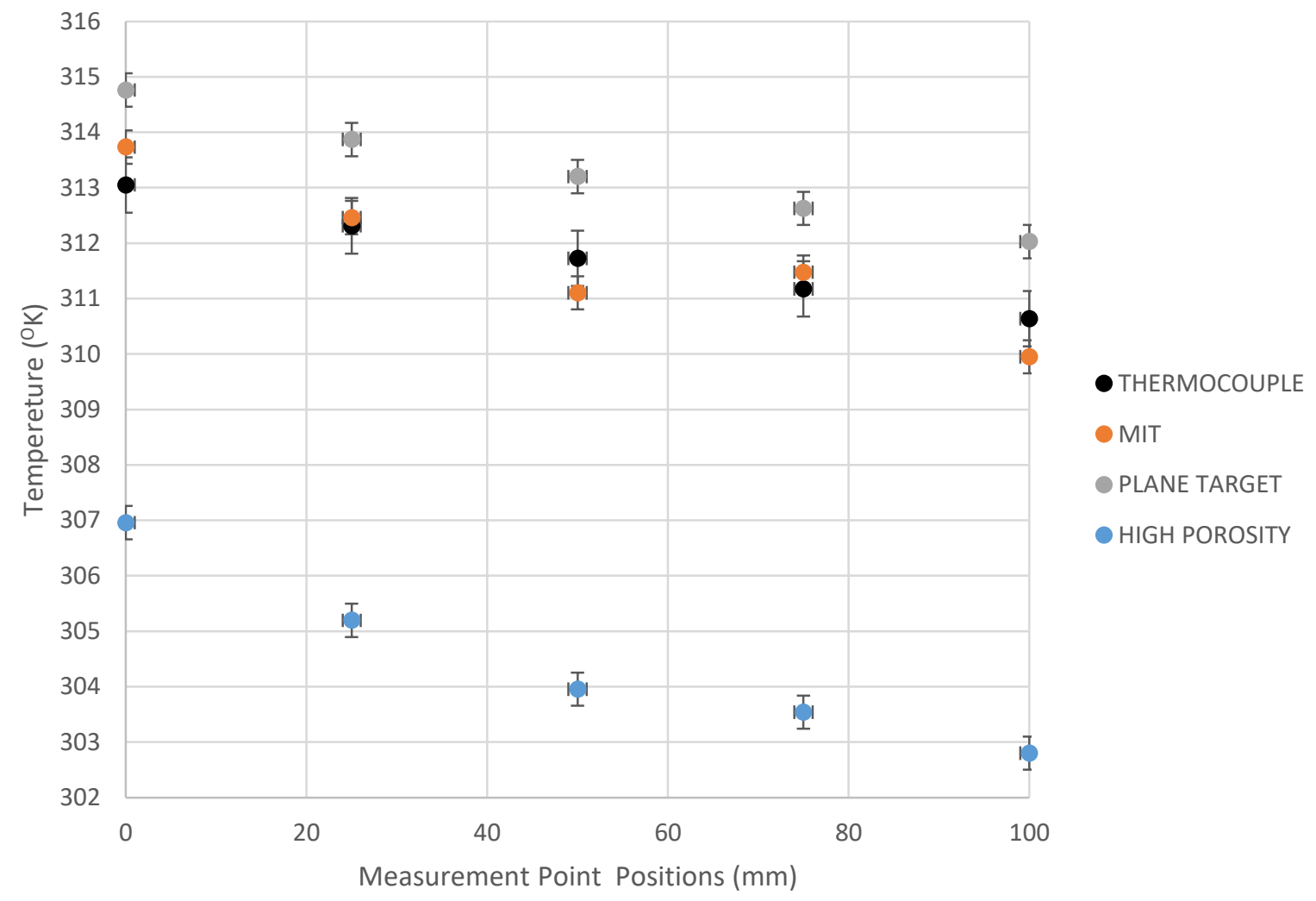

Figure 11. Comparison of the average temperature measurements obtained using the measurement screens and thermocouples.

Figure 11 shows that the results closest to those obtained via the thermocouple measurements were obtained via the MIT method. The average absolute error of the MIT results was $0.48 \mathrm{~K}$. However, the MIT results had a slight fluctuation due to image processing effects. This fluctuation can be decreased by increasing the resolution of the thermal camera or by decreasing the distance between the thermal camera and the target, which would also increase the number of measurement points on a sphere and enhance the accuracy of the results. 
After examining the trend obtained with the plane target, we observed that it measured 1.51 $\mathrm{K}$ higher at every measurement point. The reason for this is that the plane target also transfers the heat of the hot jet via conduction. Therefore, the temperature distribution becomes more diffused. This phenomenon can also be seen in Figure 12. In Figures 12-A and 12-C, the temperature distribution was not expanded downward because the jet did not affect this area. However, in Figure 12-B, the temperature distribution had expanded downward via conduction due to the plane target.

The results obtained for the high porosity target had the highest amount of absolute error, with an average of $7.29^{\circ} \mathrm{C}$. This measurement error occurred because the thin wires did not fill the pixel areas, and the results represent the area-weighted average of the thin wires and the background. The amount of error would increase if the temperature difference between the jet and the background increased.

It should be noted that, for a fair investigation, in these experiments the targets were placed parallel to the flow, which results in the minimum drag coefficient. As indicated in Section 4.1, as the angle of attack increases, the flow distortion caused by the plane target and the high porosity target will also increase and deform the temperature distribution. So it can be said that if the angle of attack would be changed, 3D MIT's accuracy is not effected due to its geometry. However, the other two measurement screens accuracy would decrease dramatically.

Another important point in Figure 12 is the spatial resolution of the results. As the thermal images investigated, it can be seen that Plane Target and High Porosity Target uses the whole imaging area. However MIT only uses the sphere areas for measurement which means lesser spatial resolution. 

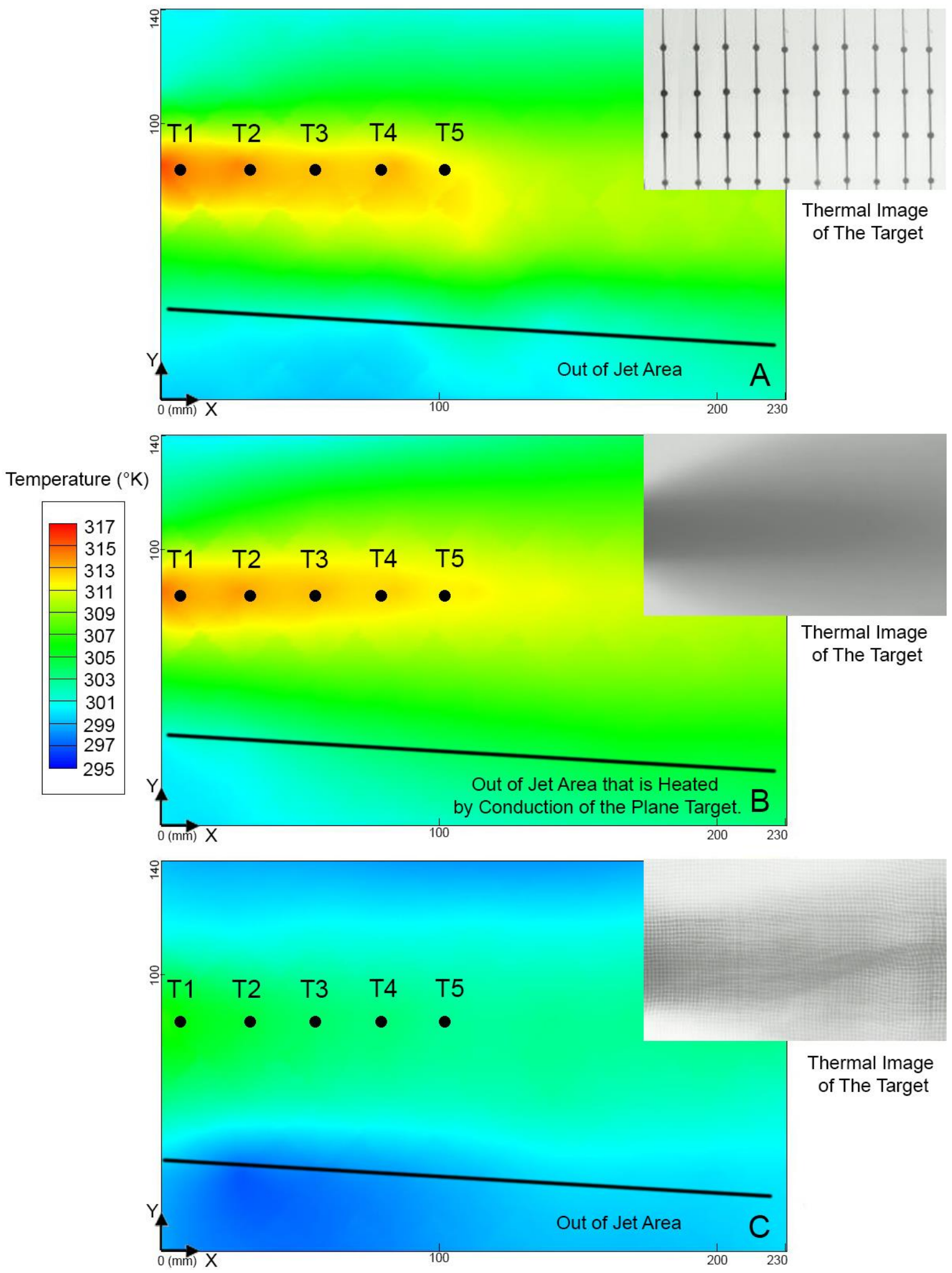

Figure 12. Comparison of average temperature distribution obtained by A) MIT, B) Plane Target, C) High Porosity Target 
As a summery, MIT target has higher accuracy and less flow deformation among the other measurement screens but also has less spatial resolution. The number of the spheres can be increase for increasing the spatial resolution however it will also increase the flow deformation as stated at section 2. On the other hand the gaps between lines is used for imaging extra planes in 3DMIT (Figure 1). So a large amount of the resolution that would be lost used and volumetric data obtain in this method with better accuracy and less flow distortion.

\section{5- 3D flow structure validation of 3D MIT via PIV}

For our final analysis of the results obtained via 3D MIT, the data obtained from the measurement mesh was used for reconstruction of the volumetric temperature distribution. At the first step of this process, the temperatures of the 315 spheres in the thermal image of the target were matched to the positions of the spheres in three-dimensional space. This matching can be done either manually or via automated algorithms by taking advantage of the fixed geometry of the measurement target. Then, the measured temperatures were positioned in virtual 3D media for visualizing. To get a clearer view, isotemperature surfaces were calculated and are shown in Figure 13-A. In Figure 13-B, the average volumetric velocity isosurfaces were reconstructed using the SPIV method to validate and compare the flow structure. To acquire this data, 16 equally spaced measurement planes $(15 \mathrm{~mm}$ intervals through $0 \mathrm{~mm}$ to $180 \mathrm{~mm}$ ) were selected throughout the outlet of the CFF, and the SPIV experiments were carried out in these planes. Then, two-dimensional three-component velocity maps were obtained for each measurement plane. The gathered data planes were placed sequentially within a three-dimensional space in a virtual environment and interpolated to form the average three-dimensional flow field of the CFF. 


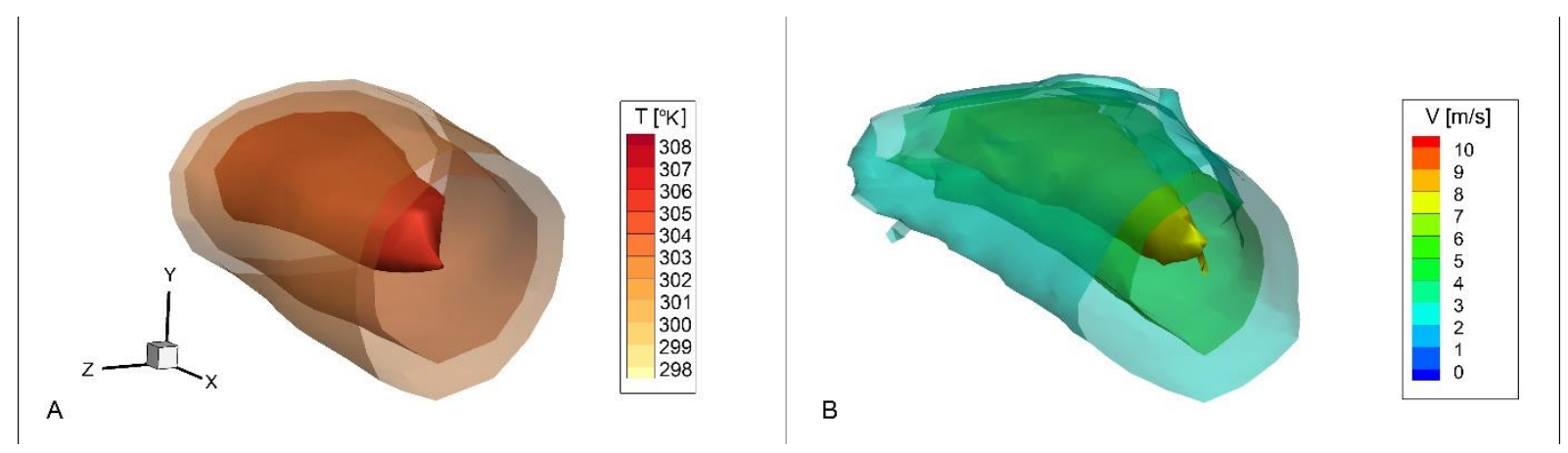

Figure 13. A) Average volumetric temperature isosurfaces of the CFF obtained via 3D MIT. B) Average volumetric velocity isosurfaces of the CFF obtained via PIV.

The 3D MIT method measures temperature, while SPIV measures velocity. Thus, it can be said that a quantitative comparison cannot be made between these results. However, when a hot jet is blown into stationary air, the jet flow loses both its temperature and velocity because it mixes with the surrounding air. Therefore, the temperature and velocity isosurface measurements would be similar to each other in a geometrical sense. In Figures 13-A and 13$B$, this phenomenon can be seen.

Based on all these results, it can be said that the 3D MIT method can be effectively used for investigating 3D flow characteristics.

\section{6- Conclusion}

The advantages and disadvantages of the 3D MIT method compared with other measurement screens were scrutinized.

The most important advantage of the 3D MIT method is that the surface area of the target is sufficiently small to be minimally affected by environmental radiation influences, but is also large enough to allow for accurate temperature measurements with a thermal camera. Therefore, the 3D MIT method has less error rate than other measurement screens. The temperature distribution that was obtained using the spheres on the thin suspension ropes with a low heat-transfer coefficient more accurately represents the temperature distribution of the fluid compared to the other measurement screens. In other words, the 3D MIT method is less affected by heat conduction. 
The 3D MIT target has low flow-blocking effects owing to the small target volume placed at the flow area. Therefore, the number of points measured per temperature plane decreases, but the other planes can be imaged from the spaces between them. Thus, simultaneous threedimensional temperature measurements with a single camera can be performed. Other measurement screen methods do not allow for simultaneous volumetric temperature measurements because their geometries block the necessary views.

As it is mentioned before, 315 points were measured in the present method. Various difficulties will arise if the same amount of measurement points were investigated by thermocouples. The most important problem is the acquisition and management of a large number of thermocouples and the dataloggers to read the data from them. A very attentive study must be done to avoid mixing the positions of the thermocouples. Another difficulty is placing the thermocouples in the flow field and keeping them in a fixed position. Even if all this is accomplished, 315 thermocouples will create an obstacle to the flow so that will prevent accurate measurement.

Scanning with one probe or a thermocouple is also an accepted method. However, it is very time consuming and it does not allow simultaneous investigation of the whole flow field. Method of scanning is also important and it can cause different problems. There are two types of scanning methods. Manual scanning is the most common method, but the position uncertainty is very high and it is vulnerable to user errors. Scanning by a traverse system is another alternative scanning method which has a capability of precise positioning. On the other hand, it is expensive and it has limited practical use in the field. There are a lot of situations that both scanning method cannot be used. Investigation of HVAC channel or heated volume of an oven can be examples to these situations. The closed cavity prevents an operator using her hand, and a traverse is generally too heavy and big to fit inside. Also, the traverse inside of a closed cavity creates a greater thermal inertia than the suggested method.

If a non-steady state flow will be investigated, the thermal inertia is critical for all the measurement screen methods. As the mass of the measurement screen and the materials' thermal capacity of the measurement screen increases the thermal inertia effect also 
increases. Among the measurement screens, 3DMIT measurement screen has a lower thermal inertia effect due to its smaller mass.

The presented state of the system can be used to study steady-state flows. However, it is also possible to investigate different flow structures with the changes and calculations expressed in Sections 2 and 2.1.

The proposed method may be utilized in many situations involving volumetric temperature distribution measurements of infrared-transparent flows (air, gases, etc.). The obtained temperature distributions can be advantageous for R\&D applications and product development. The portability and ease of implementation of the proposed system allows for both laboratory and field use. The method has already begun to be tested in design development processes in an air conditioner and an oven factory. As the method is developed, it is expected to find a wider application area.

In ongoing work, more accurate results could be obtained by optimizing the placement of the spheres forming the 3D MIT target according to the flow. This method is also being evaluated by testing it in various fields of application and comparing it with alternatives.

\section{REFERENCES}

Adrian R J (2005) Twenty years of particle image velocimetry. Experiments in Fluids 39:159169.

Adrian R J, Westerweel J (2011) Particle image velocimetry. Cambridge University Press.

Anderson R, Hassani V, \& Kirkpatrick A (1991) Visualizing the Air Flow from Cold Air Ceiling Jets. ASHRAE Journal 33:30-35.

Arlit M, Partmann C, Schleicher E, Schuster C, Hurtado A, Hampel U (2018) Instrumentation for experiments on a fuel element mock-up for the study of thermal hydraulics for loss of cooling or coolant scenarios in spent fuel pools. Nuclear Engineering and Design 336: 105-111.

Bruchhausen M, Guillard F, Lemoine F (2005) Instantaneous measurement of two-dimensional temperature distributions by means of two-color planar laser induced fluorescence (PLIF). Experiments in Fluids 38:123-131. 
Burch S D, Hassani V, \& Penney T R (1992) Use of Infrared Thermography for Automotive Climate Control Analysis. Warrendale: National Renewable Energy Laboratory.

Cehlin M, Moshfegh B, Sandberg M, (2002) Measurements of Air Temperatures Close to a Low-Velocity Diffuser in Displacement Ventilation Using an Infrared Camera. Energy and Buildings 34:687-698.

Dabiri D (2009) Digital particle image thermometry/velocimetry: A review. Experiments in Fluids 46:191-241.

Eck B (1973) Design and operation of centrifugal, axial flow and cross flow fans. Pergamon Press, Oxford.

Einecke S, Schulz C, Sick V (2000) Measurement of temperature, fuel concentration and equivalence ratio fields using tracer LIF in IC engine combustion, Applied Physics B 71:717723.

Elvsén P, Sandberg, M (2009) Buoyant Jet in a Ventilated Room: Velocity Field, Temperature Field and Air Flow Patterns Analysed with Three Different Whole-Field Methods. Building and Environment, 44:137-145.

Gallo M, Kunsch J P, Rösgen T (2010) A Novel Infrared Thermography (IRT) Based Experimental Technique for Distributed Temperature Measurements in Hot Gas Flows. 10th International Conference on Quantitative Infrared Thermography, Quebec.

Hirasawa K, Kojima H, Tomimura T (2015) Proposal of simple measurement method for peak detection capability of microscopic area by infrared thermograph. Transactions of the Japan Institute of Electronics Packaging, 8-1.

Incropera F P, DeWitt D P (1996) Fundamentals of heat and mass transfer (Fourth Edition b.). New York: John Wiley \& Sons.

Jacobson R E (1978) The Manual of photography: Formerly the Ilford manual of photography (Seventh Edition). Focal Press, London.

Karadeniz Z H, Kumlutaş D, Özer Ö (2013) Experimental visualization of the flow characteristics of the outflow of a split air conditioner indoor unit by meshed infrared thermography and stereo particle image velocimetry. Exp. Therm. Fluid Sci. 44:334-344.

Kelm S, Ritterath M, Prasser H M, Allelein H J (2016) Application of the MiniPanda test case 'erosion of a stratified layer by a vertical jet' for CFD validation. Nuclear Engineering and Design 299: 124-135.

Lazzarotto L, Lazzarotto A, Martegani A D (2001) On cross-flow fan similarity: effects of casing shape. J. Fluids Eng. 123:523-531. 
Lomperski S, Gerardi C, Lisowski D. (2016) Fiber optic distributed sensors for high-resolution temperature field mapping. J Vis Exp. 117: 54076.

Miles R, Lempert W (1990) Two-dimensional measurement of density, velocity, and 25 temperature in turbulent high-speed air flows by UV Rayleigh scattering. Appl. Phys. B Photophysics Laser Chem. 51:1-7.

Minkina W, Dudzik S (2009) Infrared thermography: errors and uncertainties. UK: Wiley, West Sussex.

Neely A J (2008) Mapping Temperature Distributions in Flows Using Radiating High-Porosity Meshes. Experiments in Fluids 45: 423-433.

Neto L P, Gamerio Silva M C, Costa J J (2006) On the Use of Infrared Thermography in Studies with Air Curtain Devices. Energy and Buildings 38: 1194-1199.

Özer Ö, Kumlutaş D (2017) Experimental investigation on cross flow fan's casing parameters inside of a split air conditioner indoor unit by Stereo Particle Image Velocimetry. Applied Thermal Engineering 124:1233-1246.

Raffel M, Willert C, Wereley S, Kompenhans J (2007) Partical Image Velocimetry: a Pratical Guide (2nd. ed.). Springer, Berlin Heidelberg New York.

Ritterath M, Öztürk O C, Prasser H M (2011) Thermo-resistive mesh sensors (TMS) for temperature field measurements. Flow Measurement and Instrumentation 22(4):343-349.

Sakakibara J, Adrian R J (1999) Whole field measurement of temperature in water using twocolor laser induced fluorescence. Experiments in Fluids 26:7-15.

Schafer T, Schubert M, Hampel U (2013) Temperature grid sensor for the measurement of spatial temperature distributions at object surfaces. Sensors 13(2): 1593-1602.

Vinnichenko N, Plaksina Y, Yakimchuk O, Soldatenkova K, Uvarov A (2016) Air flow temperature measurements using infrared thermography. Quantitative InfraRed Thermography Journal 1-15.

\section{Figure Captions}

Figure 1. Three-dimensional meshed infrared thermography setup.

Figure 2. A) Target projection on the sensor and B) simulated temperature map.

Figure 3. Optical schematic of the proposed 3D MIT setup. 
Figure 4. The effect of sphere diameters to the time required for a $0.5 \mathrm{~K}$ heating of a sphere at $1 \mathrm{~K}$ temperature difference.

Figure 5. The schematic of the experimental setups. A) 3D MIT experimental setup B) 2D measurement screens experimental setup

Figure 6. Linear regression fitting between the measurements of both validation experiments.

Figure 7. PIV comparison of the measurement screens, which were placed parallel to the jet flow. a) Results without a measurement screen, and results using b) the MIT target, c) the plane target, and d) the high porosity target.

Figure 8. Comparison of different measurement screens' flow profiles obtained by PIV (The measurement screens are placed parallel to the jet flow.)

Figure 9. PIV comparison for measurement screens placed at an angle of 30 degrees with the flow. a) Results without a measurement screen, and results b) with the MIT target, c) the plane target, and d) the high porosity target.

Figure 10. Comparison of different measurement screens' flow profiles obtained by PIV (The measurement screens are placed at an angle of 30 degrees with the flow)

Figure 11. Comparison of the average temperature measurements obtained using the measurement screens and thermocouples.

Figure 12. Comparison of average temperature distribution obtained by A) MIT, B) Plane Target, C) High Porosity Target

Figure 13. A) Average volumetric temperature isosurfaces of the CFF obtained via 3D MIT. B)Average volumetric velocity isosurfaces of the CFF obtained via PIV.

\section{Table Captions}

Table 1. Dimensions and specifications of the 3D MIT target that is used in the current study 